\title{
Generation of lossy mode resonances by deposition of high- refractive-index coatings on uncladded multimode optical fibers
}

\author{
I Del Villar ${ }^{1}$, C. R. Zamarreño, P. Sanchez, M. Hernaez, C. F. Valdivielso, F. J. \\ Arregui and I. R. Matias \\ Electrical and Electronic Engineering Department, Public University of Navarra, \\ 31006 Pamplona, Spain \\ E-mail: ignacio.delvillar@unavarra.es
}

\begin{abstract}
A comparative study of lossy mode resonances generated by depositing two different materials is presented. The two materials selected are Indium Tin Oxide (ITO) and Indium Oxide. Both materials present different dielectric dispersion, which leads to the generation of single-peak lossy mode resonances with ITO coated optical fibers and dual-peak lossy mode resonances with the $\mathrm{In}_{2} \mathrm{O}_{3}$ coated optical fibers. The obvious advantage of a dual peak based measurement in the sensors field is enhanced by a sensitivity increase observed in sensors based on $\mathrm{In}_{2} \mathrm{O}_{3}$ if compared with those based on ITO. These characteristics are analyzed both theoretically and experimentally.
\end{abstract}

\section{Introduction}

The fabrication of optical fiber sensors based on deposition of nanocoatings has experienced an exponential growth in the past years [1-5]. Most of the designs are based on two main ideas. The first one is the deposition of a coating on a cleaved optical fiber tip [6,7]. The second design consists of depositing the coating on the cladding of the optical fiber, which has been successfully proved in longperiod fiber gratings [8], tapers [9], hollow core fibers [10], or even combinations of these technologies [11]. But probably the most widely explored design within those based on coating the cladding of the optical fiber, has been the deposition of coatings on uncladded multimode fiber [12]. The surface plasmon resonance (SPR) generated by coupling of evanescent light to a surface plasmon polariton is used as the sensing mechanism. This SPR can be observed in the transmission spectrum when metallic materials (typically silver and gold $[12,13]$ ) are deposited on the cladding of an optical fiber. Recently it has been possible to obtain similar results with a transparent conductive oxide: Indium Tin Oxide [14].

The conditions that must satisfy absorbing thin-films for supporting a surface plasmon polariton and hence an SPR are described in [15]. Basically, the real part of the thin-film permittivity must be negative and higher in magnitude than both its own imaginary part and the permittivity of the material surrounding the thin-film (i.e. the optical waveguide and the surrounding medium in contact with the thin-film). However, when the real part of the thin-film permittivity is positive and higher in

1 To whom any correspondence should be addressed. 
magnitude than both its own imaginary part and the material surrounding the thin-film, a different mode is supported. Some authors consider these modes as long-range guided modes [15], whereas others call them lossy modes (LM) [16,17]. In this work the latter notation (lossy modes) will be used, and the resonance obtained by coupling to a LM will be lossy mode resonance (LMR). Light propagation through semiconductor cladded waveguides has been studied in $[18,19]$, where for specific thickness values, attenuation maxima of the light propagating through the optical waveguide are obtained [18]. This is due to coupling between a waveguide mode and a particular lossy mode of the semiconductor thin-film, which depends on two conditions: there is a considerable overlap between the mode fields, and the phase-matching condition (i.e. the equality of real parts of propagation constants) is sufficiently satisfied [16]. Since the phenomenon occurs when the lossy mode is near cut-off, it is stated in [18] that there are mode cut-off thickness values that lead to attenuation maxima. The same phenomenon can be observed if the variable is the wavelength and not the thickness. If the thin-film thickness is fixed, a resonance will be visible in the electromagnetic spectrum for those incident wavelength values where there is a mode near cut-off in the overlay. This has been proved theoretically and experimentally in [14], where the transmission spectrum of the light propagated through an Indium Tin Oxide (ITO) coated optical fiber is characterized by one or several LMR. The central wavelength of the LMR is shifted if the surrounding refractive index is modified. Consequently, it can be used as a refractometer. Moreover, a humidity sensor has been also developed based on the same technology [20].

One of the main differences with SPR is that LMR can be observed both for TE and TM polarized light [15]. However, in [14] this idea can be only understood theoretically, because in the experimental setup the light source is a white light source and because light is propagated through a multimode fiber. As a result the light that is propagated through the thin-film coated region is unpolarized, and it is not possible to analyze the polarization. Another important difference with SPR technology is that if the coating thickness is increased several attenuation bands caused by LMR can be obtained in the transmission spectrum, which does not occur with SPR based devices. Along with the thickness increase, there is a reduction in the sensitivity of the device (wavelength shift per variation of the parameter to detect) [14].

In this work, Indium Oxide $\left(\operatorname{In}_{2} \mathrm{O}_{3}\right)$ has been selected as the material for the multimode optical fiber coating. The results obtained with this material are compared to those obtained with ITO. $\operatorname{In}_{2} \mathrm{O}_{3}$ presents a higher refractive index than ITO, which shows two main advantages: the sensitivity of the device is increased and the two polarizations of light can be observed in a dual-peak LMR (one peak for each polarization) instead of a single-peak LMR (the two polarizations overlap). The dual-peak permits to obtain more accurate measurements.

\section{Experimental section}

\subsection{Device fabrication}

The device developed in this work consists of a multimode optical fiber FT200EMT, purchased from Thorlabs Inc. (core diameter $200 \mu \mathrm{m}$, cladding diameter $225 \mu \mathrm{m}$, full acceptance cone $46^{\circ}$ ). The cladding of this optical fiber is removed in a portion of several centimetres in order to deposit a thinfilm, which will support one or more lossy modes. After cladding removal the optical fibers were cleaned as described elsewhere [21]. Two types of devices were fabricated. In the first one a $0.1 \mathrm{M}$ ethanol based solution of indium(III) chloride and tin(IV) chloride pentahydrate with purity $99.999 \%$ and $99.9 \%$ respectively was used. In and $\mathrm{Sn}$ ions were adjusted with a ratio of 90:10. In the second type of device a $0.1 \mathrm{M}$ indium(III) chloride solution in ethanol was used in the dip-coating fabrication process. The build up process consisted of fabrication of a multilayer structure. The deposition of each layer consisted of immersing the fiber in the solution $(1 \mathrm{~min})$, followed by a withdrawal at a constant speed rate $(4 \mathrm{~cm} / \mathrm{s})$, and a later thermal treatment at $500{ }^{\circ} \mathrm{C}$ during $30 \mathrm{~min}$. This process was repeated up to the desired coating thickness. A SEM image of the deposition of both materials (ITO and $\operatorname{In}_{2} \mathrm{O}_{3}$ ) 
on optical fiber is shown in figure 1. All the chemicals used were purchased from Sigma-Aldrich Inc. and all the measurements were performed at room temperature $\left(25^{\circ} \mathrm{C}\right)$.

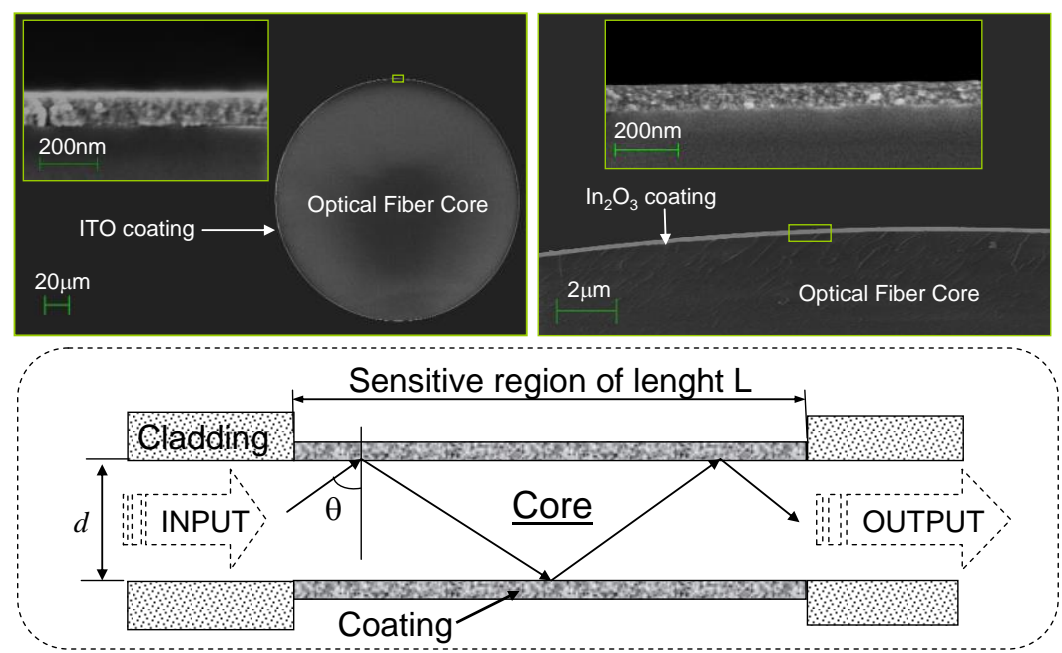

Figure 1. Top left: SEM image of an ITO coated optical fiber core with coating thickness $115 \mathrm{~nm}$ Top right: SEM image of an $\mathrm{In}_{2} \mathrm{O}_{3}$ coated optical fiber core with coating thickness $84 \mathrm{~nm}$. Bottom: Detail of the sensitive region.

\subsection{Device characterization}

The transmission spectra are obtained using the experimental setup shown in figure 2. It consists of a white light source (Ando AQ-4303B) connected to one of the optical fiber pigtails. The other one is connected to the single extreme of a bifurcated optical fiber placed to perform the simultaneous measurement of the transmitted optical power in two spectrometers: the HR4000 and the NIR512 (both from Oceanoptics Inc.). This allows covering a wide range of wavelengths, from 500 to 1700 $\mathrm{nm}$. In order to observe the wavelength shift of the LMR absorption peaks, the sensitive fragment is immersed in different glycerol in water solutions: $0 \%, 10 \%, 20 \%, 30 \%$ and $40 \%$; which respectively correspond with refractive index values: $1.321,1.335,1.35,1.365$ and 1.38. [22-23]. These values were estimated at $25^{\circ} \mathrm{C}$ and $1293 \mathrm{~nm}$. No bending curvaturte is included to avoid the influence of an additional parameter in the results.

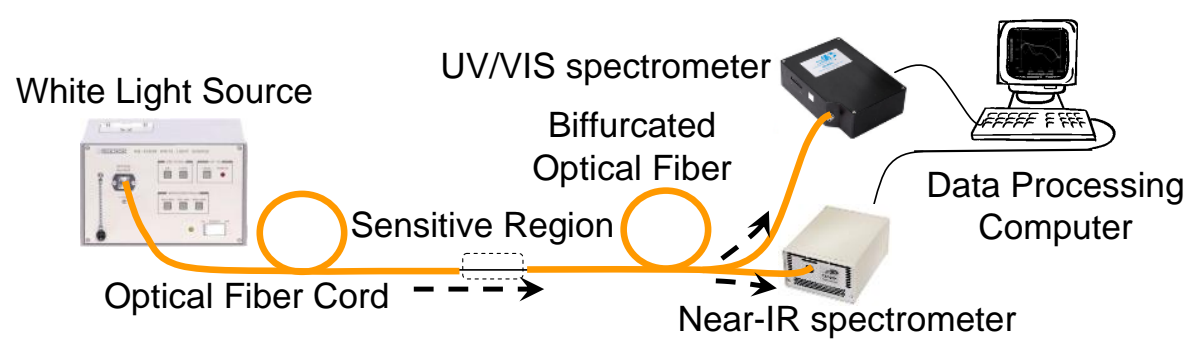

Figure 2. Experimental setup with the light source, the detector and the optical fiber with the coated region.

\section{Theory}


There are several models both for the dispersion curves of ITO and $\operatorname{In}_{2} \mathrm{O}_{3}$ and for the analysis of the propagation of light through a thin-film coated optical fiber. In this section the models used for the simulations presented in section 4 are described.

\subsection{Propagation of light through a thin-film coated optical fiber}

For the simulation of the experimental setup of figure 2, a theoretical model was developed based on the reported in other works focused on SPR phenomenon [12,24]. The applicability of this method to LMR based sensors was proved successfully in [14] for ITO coated optical fiber. In order to obtain the transmitted optical power it is important to apply first the attenuated total reflection (ATR) method with a Kretschmann configuration [25]. With this method the reflectivity as a function of wavelength and incidence angle is obtained at the coating - fiber core interface $\mathrm{R}(\theta, \lambda)[24]$.

Depending on the length of the thin-film coated region and on the incidence angle the number of reflections $N$ at the coating - fiber core interface is obtained:

$$
N(\theta)=\frac{L}{d \tan \theta}
$$

where $L$ is the length of the of the thin-film coated region, $d$ is the diameter of the optical fiber core and $\theta$ the angle of incidence.

The final step is to calculate the transmitted power. According to [24], depending on the sensor's application, the propagation of light can be analyzed considering remote sensing or nonremote sensing. Since the dimension of the fiber used in our experiments is short, it will be considered the nonremote case. The main issue now is to select an adequate equivalent of the light source power distribution $p(\theta)$ in the following expression used to calculate the transmitted power $[12,24]$ :

$$
T(\lambda)=\frac{\int_{\theta_{c}}^{90^{\circ}} p(\theta) R^{N(\theta)}(\theta, \lambda) d \theta}{\int_{\theta_{c}}^{90^{\circ}} p(\theta)}
$$

where $\theta_{\mathrm{c}}$ is the critical angle. The broadband light source was modelized with a Gaussian distribution according to [12]. Consequently, $p(\theta)$ used in expression (2) is:

$$
p(\theta) \propto \exp \left[-\frac{(\theta-\pi / 2)^{2}}{2 W^{2}}\right]
$$

where $\theta$ is the angle represented in figure 1 and $W$ indicates the width of the Gaussian function. In the simulations of section $4, \mathrm{~W}^{2}$ will be $0.075 \mathrm{rad}^{2}$.

It is also important to mention that since the light introduced in the optical fiber is unpolarized, $R^{N(\theta)}(\theta, \lambda)$ can be replaced in expression (2) with the following expression, which considers the reflected light as a combination of the reflected power at TE and TM mode polarization [26]:

$$
R^{N(\theta)}(\theta, \lambda)=\frac{R_{T M}^{N(\theta)}(\theta, \lambda)+R_{T E}^{N(\theta)}(\theta, \lambda)}{2}
$$

\subsection{Coating layer model}

According to [14] the expression used for ITO modelization is the Drude model: 


$$
\varepsilon(\omega)=\varepsilon_{\infty}-\frac{\omega_{p}^{2}}{\omega^{2}+i \frac{\omega}{\tau}}
$$

where $\varepsilon_{\infty}$ is the high frequency dielectric constant, $\tau$ is the electronic scattering time and $\omega_{p}$ is the plasma frequency. The parameters used for the modelization are: $\varepsilon_{\infty}=3.5, \tau=6.58 \times 10^{-15} \mathrm{~s} / \mathrm{rad}$ and $\omega_{p}$ $=1.533 \times 10^{15} \mathrm{rad} / \mathrm{s}$. The dispersion curves of index of refraction and extinction coefficient are represented in figure 3a. However, for the modelization of $\operatorname{In}_{2} \mathrm{O}_{3}$ a more complex expression with an additional oscillator is needed [27]:

$$
\varepsilon(\omega)=\varepsilon_{\infty}-\frac{\omega_{p}^{2}}{\omega^{2}+i \frac{\omega}{\tau}}+\frac{s_{0} \omega_{0}^{2}}{\omega_{0}^{2}-\omega^{2}+i \gamma \omega}
$$

where $s_{0}$ is the oscillator strength, $\omega_{0}$ the oscillator resonance frequency, and $\gamma$ the damping constant. The parameters used for the modelization are: $\varepsilon_{\infty}=3.5, \tau=1.014 \times 10^{-14} \mathrm{~s} / \mathrm{rad}, \omega_{p}=1.02 \times 10^{15} \mathrm{rad} / \mathrm{s}, s_{0}$ $=0.7, \omega_{0}=7.29 \times 10^{15} \mathrm{rad} / \mathrm{s}$ and $\gamma=7.08 \times 10^{14} \mathrm{rad} / \mathrm{s}$. The dispersion curves of index of refraction and extinction coefficient are represented in figure $3 \mathrm{~b}$.

a) ITO

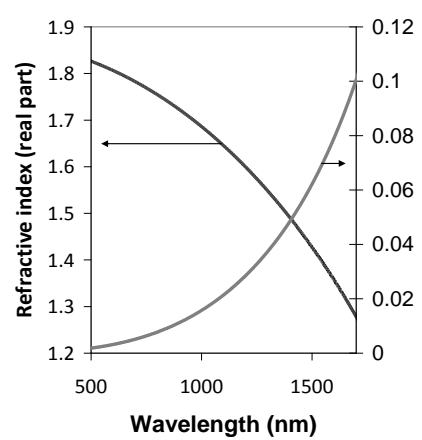

b) Indium Oxide

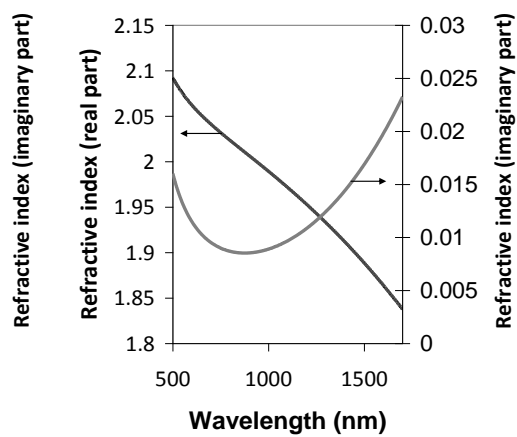

Figure 3. Real part and imaginary part of the refraction index of a) ITO deposited layer and b) $\operatorname{In}_{2} \mathrm{O}_{3}$ deposited layer

\subsection{Silica core layer model}

The refractive index of fused silica can be estimated with the well-known Sellmeier equation:

$$
n^{2}(\omega)=1+\sum_{j=1}^{m} \frac{B_{j} \omega_{j}^{2}}{\omega_{j}^{2}-\omega^{2}}
$$

with parameters: $\mathrm{B}_{1}=0.691663, \mathrm{~B}_{2}=0.4079426, \mathrm{~B}_{3}=0.8974794, \lambda_{1}=0.0684043 \mu \mathrm{m}, \lambda_{2}=0.1162414 \mu \mathrm{m}$, and $\lambda_{3}=9.896161 \mu \mathrm{m}$, where $\lambda_{\mathrm{j}}=2 \pi \mathrm{c} / \omega_{\mathrm{j}}$ and $\mathrm{c}$ is the speed of light in vacuum [28].

\section{Results}

In this section some theoretical predictions are done with the methods explained previously. The theoretical results are compared with experimental results.

Three different devices with $\mathrm{In}_{2} \mathrm{O}_{3}$ coating thicknesses of $49 \mathrm{~nm}, 86 \mathrm{~nm}$ and $174 \mathrm{~nm}$ respectively were fabricated in order to observe their spectral responses. The simulations of the transmittance spectra of 
these devices are shown in figure $4 \mathrm{~b}$ and they are compared in figure $4 \mathrm{a}$ with those obtained with ITO coating thicknesses of $115 \mathrm{~nm}, 220 \mathrm{~nm}$ and $440 \mathrm{~nm}$. For all cases it is assumed that the sensitive region is surrounded with a refractive index medium of 1.321. The experimental results obtained in figure 5 for both ITO and $\operatorname{In}_{2} \mathrm{O}_{3}$ show a qualitative agreement with the theoretical results of figure 4 .

a) ITO

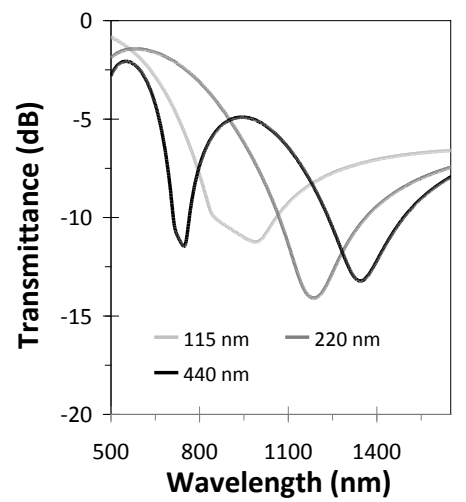

b) Indium Oxide

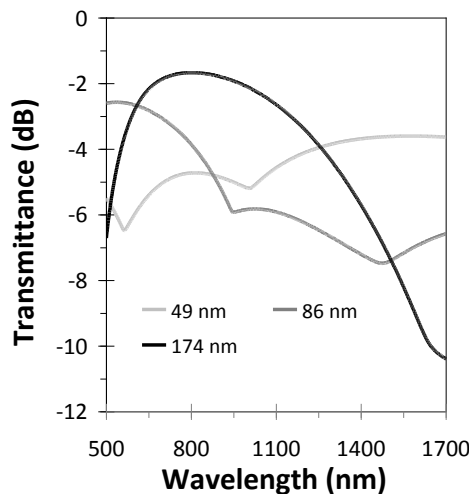

Figure 4. Simulated transmission spectra as a function of thickness for a) ITO deposited layer and b) $\mathrm{In}_{2} \mathrm{O}_{3}$ deposited layer

a) ITO

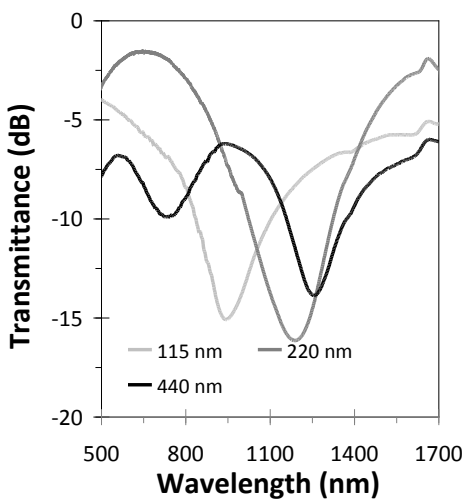

b) Indium Oxide

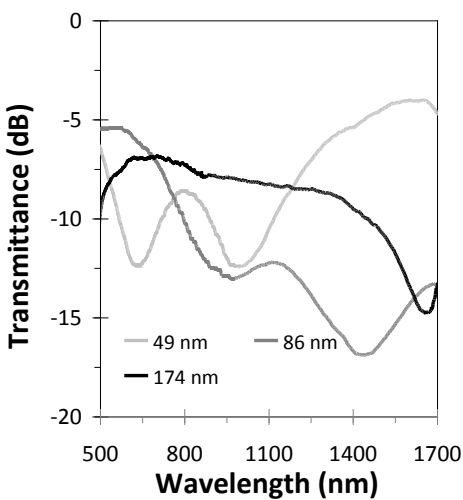

Figure 5. Experimental transmission spectra as a function of thickness for a) ITO deposited layer and b) $\operatorname{In}_{2} \mathrm{O}_{3}$ deposited layer

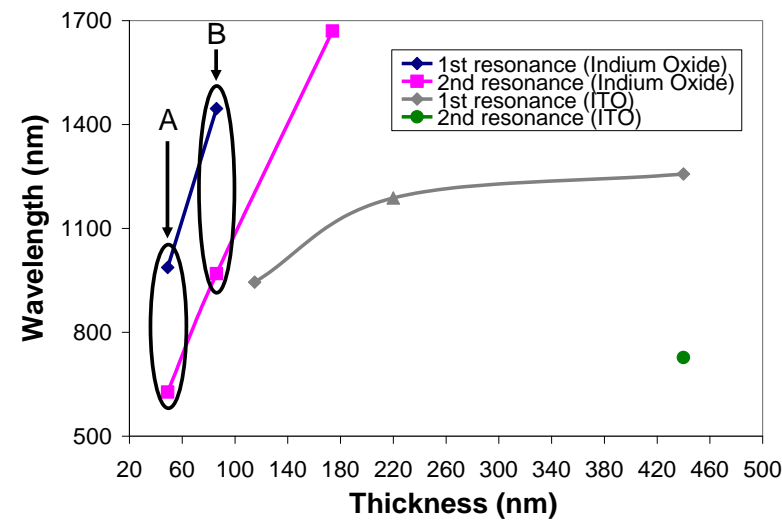


Figure 6. Resonance wavelength of ITO and $\mathrm{In}_{2} \mathrm{O}_{3}$ based devices as a function of the coating thickness when the sensitive region is immersed in ultrapure water.

In figure 6 it is presented the evolution of the central wavelength of the LMRs as a function of the thickness both for ITO and $\operatorname{In}_{2} \mathrm{O}_{3}$ coatings.

Several conclusions can be extracted from the previous results. It was indicated in the introduction that an LMR is obtained when light is coupled to near cut-off lossy modes. This leads to absorption maxima at specific thickness values [18]. Each of these maxima corresponds with a new mode that overcomes the cut-off condition and is finally guided in the coating. This idea can be extrapolated to the wavelength spectrum, where there are also attenuation bands caused by absorption maxima at specific wavelength values. Each of these attenuation bands again corresponds with a mode that overcomes the cut-off condition. Consequently, LMRs occur at specific wavelength values $[14,20]$. At high wavelengths values there is no mode guided in the coating. As we move to lower wavelength values a first mode is close to the cut-off condition, an LMR is generated in the spectrum. This LMR is considered as the first LMR because it is caused by the first lossy mode guided in the coating. As we move to lower wavelength values, a second mode overcomes the cut-off condition and is guided. As a result another LMR is obtained in the spectrum. This LMR is considered as the second LMR because it is caused by the second lossy mode guided in the coating. The same explanation is valid for the subsequent LMRs in the spectrum. To conclude, the first LMR is located at the highest wavelength and the subsequent LMRs are located at lower wavelengths. On the other hand, as the coating thickness increases, more lossy modes are guided in the coating for the same incidence wavelength. As a result, each LMR is shifted to higher wavelengths as the coating thickness is increased (see figure 6).

A second conclusion is that when $\operatorname{In}_{2} \mathrm{O}_{3}$ instead of ITO coating is used, LMRs are visible in the transmission spectrum for a lower thickness. This is logical in view of the dispersion curves of figure 3. It is well known that the condition for guidance of a mode in a waveguide is satisfied for a narrower thickness if the coating refractive index is higher [29]. That is why an $\operatorname{In}_{2} \mathrm{O}_{3}$ coating, with a higher real part of the refractive than that of ITO coating presents resonances in the transmission spectrum for a lower thickness.

Another interesting result is that the evolution of resonances as a function of coating thickness is different depending on the material used. For $\operatorname{In}_{2} \mathrm{O}_{3}$ coating the wavelength shift is linear as a function of thickness (see figure 6), whereas this is not the case for ITO coating. The explanation can be found in figure 3. The dispersion curves of $\operatorname{In}_{2} \mathrm{O}_{3}$ indicate that the condition for lossy modes is satisfied in the spectrum between 500 and $1700 \mathrm{~nm}$. However, this is not true for ITO, where the real part of the refractive index is below that of the optical fiber for wavelengths higher than $1450 \mathrm{~nm}$. At the same time, the imaginary part of the refractive index increases, which indicates that the coating could support a surface plasmon. In [14] it is explained that in this case the LMR cannot overcome the limit wavelength $(1450 \mathrm{~nm})$, which is actually observed in figure 6 .

But the most interesting aspect is the different nature of resonances obtained with $\operatorname{In}_{2} \mathrm{O}_{3}$ and ITO coatings. It is easy to observe in figure 4 that LMR are more separated for an ITO coating than for an $\mathrm{In}_{2} \mathrm{O}_{3}$ coating. For instance, when a $220 \mathrm{~nm}$ ITO coating is deposited, there is an LMR at $1188 \mathrm{~nm}$ but the second resonance is not yet visible (it is located below $500 \mathrm{~nm}$ ). However, for an $\operatorname{In}_{2} \mathrm{O}_{3}$ coating of $49 \mathrm{~nm}$, the first LMR, located $986 \mathrm{~nm}$, is already accompanied by a second LMR, located at $627 \mathrm{~nm}$. The explanation is that both LMR visible for a $49 \mathrm{~nm} \operatorname{In}_{2} \mathrm{O}_{3}$ coated device are actually the LMRs associated to two different polarizations (we group them in figure 6 as dual peak 'A'). The same is true for the $86 \mathrm{~nm} \operatorname{In}_{2} \mathrm{O}_{3}$ coated device. The two resonances observed are the LMRs associated to two different polarizations (we group them in figure 6 as dual peak 'B'). In the case of ITO coating these resonances are closer to each other and they form just a single LMR. In order to understand this question, in figure 7 it is presented the transmission spectra of two different devices (220 nm ITO coating and $49 \mathrm{~nm} \mathrm{In}_{2} \mathrm{O}_{3}$ coating) for TE polarization, TM polarization and TE+TM polarization. 
Obtaining a dual resonance is advantageous because it permits to perform dual reference measurements, with the associated improvement in accuracy. In figure 8 simulations and experimental results are shown on the wavelength shift, as a function of the surrounding medium refractive index, experimented by the dual peak ' $\mathrm{A}$ ' obtained with a $49 \mathrm{~nm} \mathrm{In}_{2} \mathrm{O}_{3}$ coated device and the dual peak ' $\mathrm{B}$ ' obtained with an $86 \mathrm{~nm} \operatorname{In}_{2} \mathrm{O}_{3}$ coated device. The sensitivity for the first peak in the $49 \mathrm{~nm}$ coated device is $3296 \mathrm{~nm} / \mathrm{RIU}$, whereas for the second one is $3847 \mathrm{~nm} / \mathrm{RIU}$. For the $86 \mathrm{~nm}$ coated device a sensitivity of $3333 \mathrm{~nm} / \mathrm{RIU}$ is obtained for the first peak whereas $4000 \mathrm{~nm} / \mathrm{RIU}$ is obtained for the second one. These results indicate that there is not a big difference between the sensitivity of the two peaks, something which was not the case for different LMRs in ITO coated devices [14]. Moreover, the sensitivity remains steady for the two coating thickness analyzed. If these results are compared to ITO coated devices where a maximum sensitivity of $1520 \mathrm{~nm} / \mathrm{RIU}$ was obtained [14], it can be concluded that the sensitivity of these devices has been improved with $\operatorname{In}_{2} \mathrm{O}_{3}$ coating by a factor greater than 2 .

a) ITO

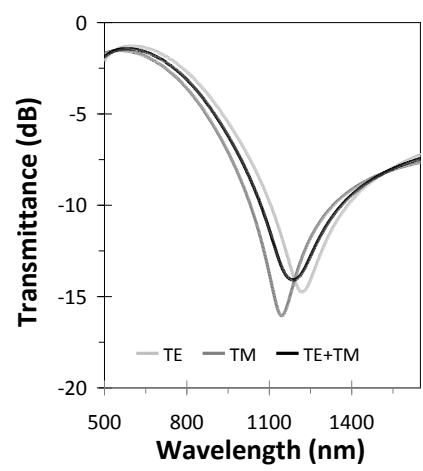

b) Indium Oxide

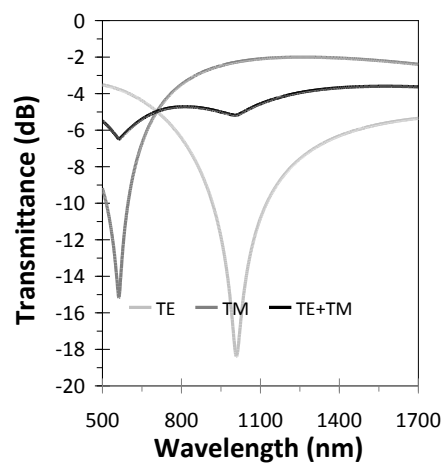

Figure 7. Simulated transmission spectra for three different polarizations: TE, TM and TE+TM. a) $220 \mathrm{~nm}$ ITO deposited layer b) $86 \mathrm{~nm} \mathrm{In}_{2} \mathrm{O}_{3}$ deposited layer

a) Simulations

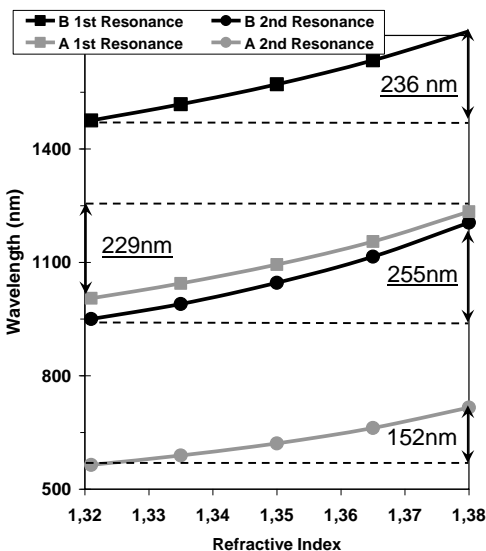

b) Experimental results

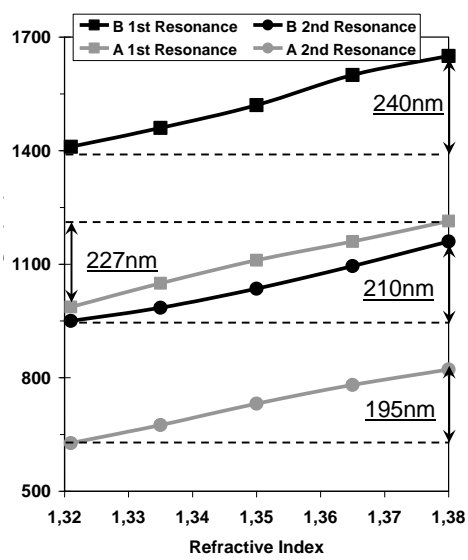

Figure 8. Simulated and experimental results of resonance wavelengths as a function of the surrounding medium refractive index for dual peak resonances obtained with $\mathrm{In}_{2} \mathrm{O}_{3}$ coatings (A indicates a $49 \mathrm{~nm}$ coating and $\mathrm{B}$ indicates an $86 \mathrm{~nm}$ coating). 


\section{Conclusions}

Lossy-mode-resonances (LMR) can be obtained in the transmission spectrum by depositing a coating on a cladding removed multimode optical fiber permits. The wavelength shift of these resonances permits to detect variations in the coating thickness, the coating refractive index or the surrounding medium refractive index.

After depositing Indium Tin Oxide (ITO) and $\mathrm{In}_{2} \mathrm{O}_{3}$ several conclusions can be extracted. First, for both materials it is clear that an LMR experiments a wavelength shift to the red if the coating thickness is increased. This can be easily explained with optical waveguide theory. Second, the dispersion curves of ITO indicate that at about $1450 \mathrm{~nm}$ the condition for LMR generation is no longer satisfied, which reduces the LMR wavelength shift as this limit is approached. This does not occur for $\operatorname{In}_{2} \mathrm{O}_{3}$ which satisfies the condition for LMR generation in the entire explored spectrum. For $\operatorname{In}_{2} \mathrm{O}_{3}$ the wavelength shift as a function of the coating thickness is linear. In addition to this, it has been proved that $\mathrm{In}_{2} \mathrm{O}_{3}$ coatings, with a higher refractive index contrast with the fiber core than ITO coatings permit to obtain dual peak resonances, which is advantageous in terms of accuracy. The explanation for this phenomenon is that the LMR observed for ITO coating is actually a combination of a TE and a TM mode. For $\mathrm{In}_{2} \mathrm{O}_{3}$ coatings, the effective indices of both lossy modes are more separated from each other and as a result two peaks belonging to each of the observed polarizations.

Finally, it has been also explored the influence of surrounding medium refractive index variations, being proved that higher refractive index coatings permit to obtain an improved sensitivity. As an example, the sensitivity obtained with ITO coated devices is improved by a factor of more than 2 for $\mathrm{In}_{2} \mathrm{O}_{3}$ coated devices.

In view of the interesting results obtained by the devices analyzed in this work, they may constitute a good platform for the analysis of other parameters such as the effect of bending the optical fiber or changing the coating refractive index.

[1] Wolfbeis OS 2002 Anal. Chem. 74 2663-2678

[2] James SW and Tatam RP 2003 Meas. Sci Technol. 14 R49-R61

[3] Lee B, Roh S and Park J 2009 Optical Fiber Technol. 15 209-221

[4] Del Villar I, Matias IR and Arregui FJ 2008 Current Analytical Chemistry 4 341-355

[5] Arregui FJ 2007 Sensors based on nanostructured materials (New York: Springer)

[6] Arregui FJ, Liu Y, Matias IR and Claus RO 1999 Sens. Actuators B 59 54-59

[7] Penza M, Cassano G, Aversa P, Cusano A, Cutolo A, Giordano M and Nicolais L 2005 Nanotechnology 162536

[8] Rees ND, James SW, Tatam RP and Ashwell GJ 2002 Opt. Lett. 27 686-688

[9] Corres JM, Bravo J, Matias IR and Arregui FJ 2006 IEEE Photon. Technol. Lett. 18 935-937

[10] Bravo J, Matias IR, Del Villar I, Corres JM and Arregui FJ 2006 J. Lightwave Technol. 24 2100-2107

[11] Pilla P, Foglia Manzillo P, Giordano M, Korwin-Pawlowski ML, Bock WJ and Cusano A 2008 Opt. Express 16 9765-9780

[12] Jorgenson RC and Yee SS 1993 Sens. Actuators B 12 213-220

[13] Yu X, Zhang Y, Pan S, Shum P, Yan M, Leviatan Y and Li 2010 J. Opt. 12015005

[14] Del Villar I, Zamarreño CR, Hernaez M, Matias IR, Arregui FJ 2010 J. Lightwave Technol. 28 111-117

[15] Yang F and Sambles JR 1997 J. Mod. Opt. 44, 1155-1163

[16] Marciniak M, Grzegorzewski J and Szustakowski M 1993 IEE Proceedings J. 140 247-251

[17] Razansky D, Einziger PD and Adam DR 2005 Phys. Rev. Lett. 95018101 
[18] Batchman TE and McWright GM 1982 IEEE J. Quantum Electron. 18 782-788

[19] Carson RF and Batchman TE 1990 App. Opt. 29 2769-2780

[20] Zamarreño CR, Hernaez M, Del Villar I, Matias IR, Arregui FJ 2010 Sens. Actuators B 146 414-417

[21] Ota R, Seki S, Ogawa M, Nishide T, Shida A, Ide M, Sawada Y 2002 Thin Solid Films, 411 4245

[22] Cooper PR 1983 Appl. Opt. 22, 3070-3072

[23] Masahiko D, Masumura A 2007 Appl. Opt. 46, 3811-3820

[24] Sharma AK, Gupta BD 2005 Opt. Comm. 245, 159-169

[25] Homola J 2006 Surface Plasmon Resonance Based Sensors (Berlin Heidelberg: Springer)

[26] Xu Y, Jones NB, Fothergill JC and Hanning CD 2000 J. Mod. Opt. 47 1099-1110

[27] Laux S, Kaiser N, Zöller A, Götzelmann R, Lauth H and Bernitzki H 1998 Thin Solid Films 335 $1-5$

[28] Agrawal GP 2001 Nonlinear fiber optics $3^{\text {rd }}$ ed. (New York, Academic Press) p 8

[29] Del Villar I, Matias IR, Arregui FJ and Lalanne P 2005 Opt. Express 13 56-69 